\title{
Liver involvement in polymyalgia rheumatica and giant cell arteritis
}

\author{
Elvis Hysa ${ }^{1}$, Alberto Castagna ${ }^{2}$, Ciro Manzo ${ }^{3}$ \\ ${ }^{1}$ Research Laboratory and Academic Division of Clinical Rheumatology, Department of Internal Medicine, San Martino Policlinic \\ Hospital, Genoa, Italy \\ ${ }^{2}$ Azienda Sanitaria Provinciale Catanzaro, Geriatric Medicine Department, Fragility Outpatient Clinic, Casa della Salute di Chiaravalle \\ Centrale, Catanzaro, Italy \\ ${ }^{3}$ Internal and Geriatric Medicine Department, Azienda Sanitaria Locale Napoli 3 sud, Rheumatologic Outpatient Clinic Hospital \\ “Mariano Lauro”, Sant’Agnello, Naples, Italy
}

\section{Dear Editor}

We read with great interest the review paper entitled "Liver involvement in rheumatic diseases" [1]. In this letter, our aim is to add a brief focus about the hepatic manifestations related to polymyalgia rheumatica (PMR) and giant cell arteritis (GCA) according to the evidences of literature. Polymyalgia rheumatica is estimated to be one of the most common inflammatory rheumatic diseases affecting older adults [2]. At least 10-16\% of PMR patients have also manifestations of GCA, whereas $40-60 \%$ of GCA patients have also manifestations of PMR. Glucocorticosteroids (GCs) are the cornerstone of their treatment [3].

Liver involvement is a recognized association in PMR and GCA, but it has not been commonly reported in published literature. Multiple studies have reported an increase of serum alkaline phosphatase (AP), with a confirm of the hepatic isoenzyme, in about $30-60 \%$ of newly diagnosed PMR and GCA patients [4-6]. Less frequent is a cytolytic pattern with elevation of alanine (ALT) and aspartate (AST) transaminases without raised ALP and gamma-glutamyltranspeptidase (gGT) - it occurred in $18 \%$ of PMR patients [6].

Other causes of liver dysfunction such as alcoholism, exposure to drugs and hepatotoxic agents, viral hepatitis were excluded for these subjects. Besides, liver enzymes correlated with systemic symptoms and with higher values of the erythrocyte sedimentation rate (ESR) and C-reactive protein (CRP) concentrations. In most cases, they normalized after GCs therapy and increased during disease relapses. A limited number of biopsies have been performed in these patients documenting different histological pictures from a normal liver architecture to fatty infiltration of hepatocytes, widening of bile canaliculi, immune infiltrates of lymphocytes and plasma cells in the peri-sinusoidal spaces and, more rarely, granulo- matous lesions or vasculitis in the hepatic artery and in portal-tract arteries [7]. Interestingly, these alterations reversed with GCs treatment.

It has been also speculated whether liver damage could have immune-mediated origins. In fact, some authors observed an increase of anti-mitochondrial autoantibodies (AMA) in up to $30 \%$ of PMR patients. However, no correlation was detected between AMA titers and inflammatory indexes, clinical state or biopsy specimens [8]. It would be interesting to understand if PMR/ GCA patients are more likely to develop a primary biliary cholangitis (PBC) compared with general population, or if AMA positivity is reactive to a possible mitochondrial damage documented in these patients by studies of electron microscopy.

In the paper of Sattar et al. [9], PMR patients were classified according to their clinical and laboratory features (girdles' pain, morning stiffness and raised inflammatory markers) after excluding other causes of polyarthritis and myopathies. However, liver biopsy-associated data were lacking for most of these patients. To the best of our knowledge, the association of PMR/GCA with biopsy-proven PBC was reported in only one case [9].

A possible link between laboratory alterations and hepatic inflammatory activity detected through positron emission tomography with total body computed tomography (PET-CT) scan has also been investigated but, surprisingly, with the normalization of liver enzymes after GCs treatment, there was a persistence of tracer uptake in patients with altered pre-treatment scans. In particular, in patients with overlapping GCA, the arterial hepatic flow was lower compared with isolated PMR patients suggesting the presence of hepatic vasculitis [10].

Finally, in a patient diagnosed with PMR having an hepatic dysfunction characterized by elevation of ALP 
and gGT (almost always without jaundice) associated with marked elevation of ESR and CRP concentrations, a paraneoplastic syndrome known as Stauffer's syndrome should be considered in differential diagnosis.

Stauffer's syndrome bas been classically described in early stage renal cell carcinoma, but it can be occasionally associated with other malignancies. Thrombocytosis and systemic manifestations are often present, while bilirubin serum levels are usually in their normal range. No intra-hepatic or extra-hepatic ductal dilatation is present when abdominal ultrasound, magnetic resonance or endoscopic retrograde cholangiopancreatography are performed. Some investigators hypothesized that interleukin 6 (IL-6) plays a central role as its serum levels are significantly high in these patients. A poor or no response to GCs is the rule, while the surgical removal of the cancer normalizes both liver function and IL- 6 serum levels and PMR clinical manifestations [11].

\section{Conclusions}

Liver involvement, especially with a cholestatic pattern, can be present in patients with PMR and GCA, and usually disappears after GCs therapy. The possibility that liver involvement can represent a risk factor for an overlapping GCA or a warning for cancer should be carefully addressed in clinical practice. Further studies are needed to understand if PMR, antimitochondrial antibodies and PBC might be linked from similar pathogenetic mechanisms or if they can coexist by chance.

The authors declare no conflict of interest.

\section{References}

1. Podgorska J, Werel P, Klapaczynski J, et al. Liver involvement in rheumatic diseases. Reumatologia 2020; 58: 289-296, DOI: 10.5114/reum.2020.99782.

2. Hysa E, Sobrero A, Camellino D, et al. A seasonal pattern in the onset of polymyalgia rheumatica and giant cell arteritis? A systematic review and meta-analysis. Semin Arthritis Rheum 2020; 50: 1131-1139, DOI: 10.1016/j.semarthrit.2020.05.023.

3. Manzo C, Camellino D. Polymyalgia rheumatica: diagnostic and therapeutic issues of an apparently straightforward disease. Recenti Prog Med 2017; 108: 221-231, DOI: 10.1701/2695.27559.

4. Leong AS, Alp MH. Hepatocellular disease in the giant-cell arteritis/polymyalgia rheumatica syndrome. Ann Rheum Dis 1981; 40: 92-95, DOI: 10.1136/ard.40.1.92.

5. von Knorring J, Wassatjerna C. Liver involvement in polymyalgia rheumatica. Scand J Rheumatol 1976, 5: 197-204, DOI: 10.3109/03009747609099905.

6. Kyle V. Laboratory investigations including liver in polymyalgia rheumatic/giant cell arteritis. Baillère's Clin Rheumatol 1991; 5: 475-484, DOI: 10.1016/s0950-3579(05)80066-4.

7. Ogilvie AL, James PD, Toghill PJ. Hepatic artery involvement in polymyalgia arteritica. J Clin Pathol 1981; 34: 769-772, DOI: 10.1136/jcp.34.7.769.

8. Sattar MA, Cawley MID, Hamblin TJ, Robertson JC. Polymyalgia rheumatica and antimitochondrial antibodies. Ann Rheum Dis 1984; 43: 264-266, DOI: 10.1136/ard.43.2.264.

9. Gagnerie F, Taillan B, Euller-Ziegler L, Ziegler G. Primary biliary cirrhosis, temporal arteritis (giant cell arteritis) and polymyalgia rheumatica in a single patient. Scand J Rheumatol 1988; 17 : 231-232, DOI: 10.3109/03009748809098790.

10. Kyle V, Wraight EP, Hazleman BL. Liver scan abnormalities in polymyalgia rheumatica/giant cell arteritis. Clin Rheumatol 1991; 10: 294-297, DOI: 10.1007/BF02208693.

11. Blay JY, Rossi JF, Wijdenes J, et al. Role of interleukin- 6 in the paraneoplastic inflammatory syndrome associated with renalcell carcinoma. Int J Cancer 1997; 72: 424-430, DOI: 10.1002/ (sici)1097-0215(19970729)72:3<424::aid-ijc9>3.0.co;2-r. 